\title{
Hipertensión arterial. La perspectiva del médico de familia
}

\section{Arterial hypertension. A family physician's perspective}

\section{Serrano-Martínez}

La carga de morbilidad de las poblaciones occidentales está encabezada por las enfermedades cardiovasculares entre las primeras causas y son, en su conjunto, la primera causa de mortalidad global en el mundo. Son muy bien conocidos los factores de riesgo en relación a los estilos de vida y a los parámetros biológicos que pueden predecir la incidencia de cualquiera de sus manifestaciones clínicas: cardiopatía isquémica, ictus, muerte súbita, enfermedad arterial periférica e insuficiencia cardiaca ${ }^{1,2}$.

Sin ninguna duda, la hipertensión arterial es el factor de riesgo cardiovascular más prevalente en la población general. Este factor de riesgo, definido como cifras iguales o superiores a 140/90 mm de Hg, o por la toma de fármacos antihipertensivos, está presente en el 50,9\% de los hombres y el 39,4\% de las mujeres entre 35 y 84 años en un estudio poblacional realizado en Navarra entre junio de 2004 y diciembre de 2005 (Estudio de riesgo vascular de Navarra). La prevalencia se incrementa con la edad y, en las personas con más de 75 años, el $85 \%$ de las mujeres y el $71 \%$ de los hombres han sido diagnosticados o pueden ser diagnosticados de hipertensión ${ }^{3}$.

De estos datos, extrapolados a toda la población de Navarra, se puede deducir que 85.610 hombres navarros y 70.624 mujeres navarras tienen cifras elevadas de presión arterial. La responsabilidad asistencial del diagnóstico de la hipertensión, del tratamiento con cambios en estilos de vida y fármacos antihipertensivos, y el control personal de la pre-

Departamento de Medicina Preventiva y Salud Pública. Universidad de Navarra. Medicina de Familia, Centro de Salud de Azpilagaña. Pamplona.
Correspondencia: masemar@gmail.com 
sión arterial, recae sobre los profesionales que trabajan en los centros de salud de Atención Primaria, habitualmente sobrecargados de otras muchas tareas. Sin embargo, es preciso considerar el enorme beneficio del control de los hipertensos en relación con los costes del tratamiento.

Uno puede sentirse tentado de hacer una búsqueda de hipertensos entre las personas que presentan una elevada edad, por la mayor prevalencia a partir de los 65 años, o que tienen otros factores de riesgo. Pero en una población general, con edades medias entre 30 y 40 años, como la que integra el estudio SUN (Seguimiento Universidad de Navarra), la incidencia por 1.000 personas-año es relativamente importante, de 8,2 y 21,8 en mujeres y hombres respectivamente ${ }^{4}$. Esto debería impulsar a la búsqueda activa de hipertensos desconocidos de cualquier edad, de los que apenas una tercera parte han sido eficazmente diagnosticados, y de éstos, solamente una tercera parte son tratados, con frecuente descontrol en este grupo.

Si no prestáramos atención suficiente a las personas con cifras casi normales de presión arterial, aquellas con cifras en la fase I de hipertensión, es decir, con presiones sistólicas entre 140 y $159 \mathrm{~mm}$ de $\mathrm{Hg}$ o con presiones diastólicas entre 90 y $99 \mathrm{~mm}$ de $\mathrm{Hg}$, estaríamos manteniendo como hipertensos a la porción de la población responsable de la mayor fracción atribuible poblacional en riesgo de muerte cardiovascular; la cuarta parte de las muertes por estas causas suceden en este estrato de la hipertensión. Por consiguiente, se debe insistir en un abordaje poblacional en el diagnóstico de este factor de riesgo, en tarea diaria y constante, con consiguientes conductas terapéuticas apropiadas.

Son muchos los ensayos clínicos realizados sobre la conveniencia del tratamiento de la hipertensión arterial, y sobre los efectos diferenciales de los diversos antihipertensivos que aconsejan en distintas situaciones el uso de alguno en particular.

Los metaanálisis publicados en 1990 por MacMahon y col y Collins y col, establecieron, entre otras cosas, que descensos de 5-6 mm de $\mathrm{Hg}$ de la presión arterial diastólica producen reducciones del $40 \%$ en ictus y $25 \%$ en infartos de miocardio, y que la relación entre la enfermedad cardiovascular y la presión arterial no tiene umbral y es positiva y continua ${ }^{5,6}$.

Idealmente, el objetivo debería ser la reducción de las cifras medias de presión arterial en la totalidad de la población. En Navarra las cifras medias de presión arterial son de 
138,4/81,6 mm de Hg en los hombres y 129,5/78,2 mm de Hg en las mujeres. Este objetivo de salud pública debe ser fruto, no sólo de actividades de protección de la salud contempladas como estrategias de gobierno, sino como una labor continuada de la actividad preventiva y de promoción de la salud en atención primaria.

Existen datos que aportan evidencias científicas sobre el consejo dietético y de actividad física para la reducción de la presión arterial, que no debería ser únicamente dado a personas hipertensas, sino también a otras en riesgo de presentar hipertensión arterial futura, tales como a pacientes con sobrepeso y/u obesos, diabéticos, con antecedentes familiares de hipertensión, y personas con cifras de hipertensión normal-alta (130-139/85-90 mm de Hg).

La dieta por sí sola es capaz de reducir de modo sustancial las cifras de hipertensión tanto en pacientes hipertensos (reducciones medias de 11,4 y 5,5 mm de $\mathrm{Hg}$ en presión sistólica y diastólica respectivamente), como en personas no hipertensas (3,5 y 2,1 mm de Hg en presión sistólica y diastólica respectivamente). El ensayo DASH (Dietary Approach to Stop Hypertension), realizado durante tres semanas con una dieta rica en verduras, frutas, lácteos desnatados y pobre en grasa total y saturada, aportó una primera evidencia del tratamiento dietético de la hipertensión arterial ${ }^{7,8}$.

En España, el estudio multicéntrico PREDIMED (Prevención con Dieta Mediterránea), en el que participan pacientes de alto riesgo cardiovascular tratados en varios centros de salud de Navarra, ha demostrado en los primeros tres meses tras la asignación aleatoria de los pacientes a dieta mediterránea enriquecida en aceite de oliva extra virgen, a dieta mediterránea enriquecida en frutos secos (avellanas, nueces y almendras), o a dieta control pobre en grasa, que no sólo la adherencia a la dieta era correcta, sino que la presión arterial descendió significativamente en los dos grupos de dieta mediterránea, mientras que no se modificó en el grupo de dieta pobre en grasa total. La reducción de la presión sistólica y diastólica respectivamente en el grupo con dieta enriquecida en aceite de oliva fue de 4,8 y 2,5 $\mathrm{mm}$ de $\mathrm{Hg}$, y en el grupo con dieta enriquecida en frutos secos fue de 6,5 y 3,6 mm de $\mathrm{Hg}$, ambos descensos significativos frente al observado en el grupo control y frente a las cifras de partida del propio grupo. Además mejoró la sensibilidad a la insulina sin cambios diferenciales en el peso ni en el perímetro de cintura ${ }^{9}$. Estas evidencias trasladadas mediante diversas estrategias a toda la 
población reducirían la incidencia de hipertensión de modo sustancial, el riesgo cardiovascular prevalente en la población general, y por consiguiente la necesidad de tratamientos farmacológicos.

Por otra parte, es también conocido el efecto de la actividad física sobre las cifras de presión arterial y sobre la morbimortalidad cardiovascular en general. Los ensayos, en general de breve duración, demuestran reducciones significativas tanto de la presión sistólica como de la diastólica ${ }^{10}$. Por otra parte, la incidencia de hipertensión se ha relacionado con el sedentarismo en una cohorte española ${ }^{11}$.

El consejo sobre estilos de vida debe ser por tanto una constante en las consultas de atención primaria, y no forzosamente ha de ser una actividad ardua y prolongada. El arma fundamental de los médicos de familia son las repetidas consultas con cada paciente, que hace más eficaz el consejo breve y repetido, con objeto de evitar la instauración de factores de riesgo y facilitar la mejoría de los ya presentes. Esta actitud mantenida puede hacer menos necesaria la escalada en las dosis de antihipertensivos o hipolipemiantes, por lo que es básicamente eficiente y con una esperada buena relación coste-eficacia.

Por último, en relación a la necesidad de medicación antihipertensiva, muy frecuentemente necesaria para mantener cifras normales a los pacientes con presión arterial no controlable por otros medios, es preciso mantener una actitud activa. Los casos de hipertensión refractaria son escasos, y la falta de control es con frecuencia debida a una ausencia de adherencia de los pacientes al tratamiento, a la falta de revisiones de la evolución de las cifras de presión arterial, o al retraso en la adecuación de los tratamientos.

Llama la atención en el estudio publicado en este número de Anales del Sistema Sanitario de Navarra las asociaciones observadas respecto al grado de control de la hipertensión arterial que se encuentran en un centro de salud de esta comunidad. Pero una de las conclusiones que más destaca en ese estudio es la asociación positiva del uso de los ARA II con el control de los hipertensos, mientras que la asociación fue negativa con el grupo de los IECA. Quizá debería examinarse cuál ha sido la dosificación de cada uno. Probablemente las presentaciones farmacéuticas pueden influir en la dosificación diaria y en la prescripción creciente que facilita el escalado de dosis, dando una visión errónea respecto de la eficacia de los grupos de antihipertensivos. No es muy aceptable a 
priori, como la experiencia demuestra, que haya fármacos claramente más eficaces que otros en toda la población de hipertensos. Lo oportuno y conveniente es conocer las dosificaciones necesarias para cada paciente de cada fármaco, las asociaciones correctas, y para aplicarlas convenientemente es obligado hacer un seguimiento próximo con controles personalizados a todos los hipertensos.

\section{BIBLIOGRAFIA}

1. OMS. World Health Report 2004. Shaping the future. Ginebra: OMS, 2003.

2. MurRaY CJL, LOPEz AD. The global burden of disease: a comprehensive assessment of mortality and disability from diseases, injuries, and risk factors in 1990 and projected to 2020. Boston: Harvard School of Public Health, 1996.

3. Viñes JJ, Diez J, Guembe MJ, González P, AmÉzqueta C, Barba J et al. Estudio de riesgo vascular en Navarra: objetivos y diseño. Prevalencia el síndrome metabólico y de los factores mayores de riesgo vascular. An Sist Sanit Navar 2007; 30: 113-124.

4. Beunza JJ, Martínez-González MA, Serrano-Martínez M, Alonso A. Incidencia de hipertensión arterial en una cohorte de graduados universitarios españoles: el estudio SUN. Rev Esp Cardiol 2006; 59: 1331-1334.

5. MacMahon S, Peto R, Cutler J, Collins R, Sortie P, Neaton J et al. Blood pressure, stroke, and coronary heart disease. Part 1. Lancet 1990; 335: 765-774.

6. Collins R, Peto R, MacMahon S, Hebert P, Fiebach NH, Eberlein KA et al. Blood pressure, stroke, and coronary heart disease. Part 2. Lancet 1990; 335: 827-838.

7. Apple LJ, Moore TJ, Obarzanek E, Vollmer WM, Svetkey lP, Sacks FM et al for de DASH Collaborative Research Group. A clinical trial of the effects of the dietary patterns on blood pressure. N Engl J Med 1997; 336: 1117-1124.

8. PICKERING TG. New guidelines on diet and blood pressure. Hypertension 2006; 47: 135-136.

9. Estruch R, Martínez-González MA, Corella D, Salas-Salvadó J, RuizGuTIÉRREZ V, COVAS MI et al, on behalf of the PREMIMED Study Investigators. Effects of a mediterranean-style diet on cardiovascular risk factors: A randomized trial. Ann Intern Med 2006; 145 : $1-11$.

10. ROBERTS CK, VAZIRI ND, BARNARD RJ. Effect of diet and exercise intervention on blood pressure, insulin, oxidative stress, and nitric oxide availability. Circulation 2002; 106: 2530-2536.

11. Beunza JJ, Martinez-Gonzalez MA, Ebrahim S, Bes-Rastrollo M, NuÑEZ J, MARTinEz JA et al. Sedentary behaviours and the risk of incident hypertension: the SUN cohort. Am J Hypertens 2007; 20: 1156-1162. 
